\title{
Test Structures for Developing Packaging for Implantable Sensors
}

\author{
E. O. Blair, A. Buchoux, A. Tsiamis, C. Dunare, J. R. K. Marland, M. E. Gray, J. G. Terry, Senior \\ Member, IEEE, S. Smith, Senior Member, IEEE, and A. J. Walton, Senior Member, IEEE,
}

\begin{abstract}
With their capacity for real time monitoring and spatial mapping, implantable sensors are becoming an increasingly important aspect of next generation precision healthcare. Microfabricated sensor systems are a popular choice, owing to their capacity for miniaturisation, repeatable mass manufacture, and numerous pre-existing sensor archetypes. Despite the drive for development, packaging these sensors for the environment within the body, as well as the implantation process itself, presents a significant challenge. This paper presents microelectronic test structures, which can be used to assess, compare, and optimise implantable packaging solutions in a standardised manner. The proposed structures are used to investigate: (i) the capacity of the material to be patterned, (ii) the permeability of the insulation material, (iii) adhesion of the encapsulant to the die, and (iv) the physical robustness of the package to implantation through a needle. They are used to characterise an example packaging strategy, using biocompatible epoxy-resin. In addition, a method of optimising the packaging performance using the test structures is presented.
\end{abstract}

Index Terms-Implantable, Microsystems, packaging, reliability, sensors, test structures.

\section{INTRODUCTION}

$\mathbf{I}$ MPLANTABLE sensors are an important tool in the shift towards precision healthcare. The ability to continuously monitor localised, key parameters or biomarkers in a patient will enable the delivery of more targeted and rapid interventions, as well as more efficient use of medical resources and treatments. With this aim in mind, new generations of sensors are being developed to fulfil this clinical need [1], [2]. The IMPACT (Implantable Microsystems for Personalised AntiCancer Therapy) project is concerned with fabricating a suite of miniaturised sensors for the spatial mapping of hypoxia in the microenvironment of a cancerous tumour [3]. Hypoxic areas of a tumour are poorly oxygenated and have a higher resis-

This work was supported by funding from the UK Engineering and Physical Sciences Research Council, through the Implantable Microsystems for Personalised Anti-Cancer Therapy (IMPACT) programme gran (EP/K034510/1). The data presented in this paper can be found at: https://doi.org/10.7488/ds/2638.

E. O. Blair was with the Institute for Integrated Micro and Nano Systems, School of Engineering, University of Edinburgh, Edinburgh EH9 3FF, U.K (Now with the Department of Biomedical Engineering, University of Strathclyde, Glasgow, G4 0NS, UK.) (ewen.blair@strath.ac.uk)

A. Buchoux is with the Institute of Multiscale Thermofluids, University of Edinburgh, Edinburgh EH9 3FF, U.K.

A. Tsiamis and S. Smith are with the Institute for Bioengineering, School of Engineering, University of Edinburgh, Edinburgh EH9 3FF, U.K

C. Dunare, J. R. K. Marland, J. G. Terry, and A. J. Walton are with the Institute for Integrated Micro and Nano Systems, School of Engineering, University of Edinburgh, Edinburgh EH9 3FF, U.K.

M. E. Gray is with The Royal (Dick) School of Veterinary Studies and Roslin Institute, University of Edinburgh, Edinburgh, EH25 9RG, UK tance to radiotherapy. This means standard radiotherapy dosing regimes may result in these radioresistant tumour subvolumes receiving inadequate radiation doses, leading to treatment failure. Hypoxia, however, is not static and areas become more or less oxygenated as the tumour grows, presenting a challenge for conventional diagnostic technologies such as computed tomography (CT) or positron emission tomography (PET) scanners, which can only provide a snapshot in time [4]-[6]. The continuous, localised monitoring of tumour oxygen levels and key biomarkers through a miniature implantable sensor, is a potentially life-saving solution. Microfabrication offers the capability to mass-produce such small sensors in a reliable and cost-effective manner [7]. Among the many challenges in developing this kind of implantable technology is packaging.

Microsystems packaging focuses on interfacing microelectro-mechanical systems (MEMS) and sensor systems with the world in a low-cost and reliable manner [8]. Typically these sensors require some area(s) of the device to be in direct contact with its surroundings, while exposed contact pads, wire bonds, and materials incompatible with the environment are protected [9]-[11]. This is especially important with technology destined for the human body, where failed insulation means not only a potential health hazard, but readings from a faulty sensor can impact medical diagnoses. Although examples of successful commercial implantable device packaging exist, such as ceramic or metal housing for pacemakers, this is generally incompatible with the miniaturisation required for next generation sensors [12]. In addition, electrochemical sensors present an extra challenge in that a voltage must usually be applied between parts of the sensor and the external solution, which can accelerate insulation failure [13]. Numerous packaging solutions have been reported for these sensors, but are typically not available in a standardised way, such as is the case for microelectronic integrated circuits [9], [14]-[16]. Even fewer examples are available for implantable systems [10], [13], [17], [18]. The majority of implantable packaging solutions in literature are bespoke and use a large variety of different materials, processes, and designs.

Using the IMPACT sensor as a template, the key parameters for an implantable packaging system can be summed up as follows:

- biocompatibility;

- ability to be patterned to expose sensing areas;

- compatibility with post-fabrication assembly such as wire bonding; 
- the permeability or barrier properties to the surrounding environment;

- adhesion of the encapsulation material to the surface of the die, and;

- physical robustness to implantation, for example through a needle.

Microelectronic test structures offer a route to developing a system which meets these needs. Test structures enable the probing of individual parameters and can therefore identify the location of faults, facilitate systematic optimisation, and yield understanding of the separate parts of a system [19]. They are therefore ideally suited to develop an implantable packaging system by designing test structures to target each of the parameters described above. Such a systematic approach is needed in an area with stringent regulatory criteria [10], [18], [20]. It is therefore especially important to be able to thoroughly characterise packaging materials and processes for biomedical applications.

This article presents an expanded version of the ICMTS conference paper "Test Structures for the Characterisation of Sensor Packaging Technology" [21]. Four test structures are described which were used to develop the packaging for the implantable sensors developed in the IMPACT project, demonstrating their general applicability for in vivo microsensor development. A UV-curable epoxy-resin (Epo-Tek OG11631/1LB) with ISO standard 10993 biocompatibility was chosen for the sensor encapsulation. Since biocompatibility is usually established in healthy tissue, the IMPACT project has also demonstrated the biocompatibilty of sensor and packaging materials in tumour animal models [22]. The test structures were therefore used to characterise the resin's capability to be patterned, its permeability to a liquid environment, its adhesion to the die surface, and its robustness to being implanted through a needle using a sheep cadaver model. Finally, the development of an improvement to the packaging system is described, using a combination of the test structures presented, to demonstrate their capacity for optimisation.

\section{TEST STRUCTURE PHILOSOPHY AND DESIGN}

The four test die layouts have been designed with the purpose of characterising specific aspects of the packaging material and process:

1) The layout of test structure one (TS1) is presented in Fig. 1(a). It consists of a die with four rows of connected aluminium bond pads. Optical measurement of the blank central area can be used to confirm that no residue has been left behind by the patterning process, while the bond pads are used for checking electrical continuity throughout the packaging process. Schematic representations of these measurements are shown in Fig. 2(a)(i) and (ii) respectively.

2) The second test structure (TS2) layout is shown in Fig. 1(b). This uses a $2 \mathrm{~mm}$ square electrode in the centre to quantify pinhole density and/or permeability of the packaging material to its environment. This measurement set up is depicted in Fig. 2(b).

3) Test structure three (TS3) is shown in Fig. 1(c) and comprises six parallel electrodes of differing lengths. (a) TS1

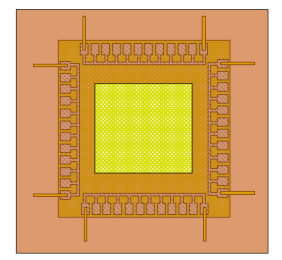

(c) TS3

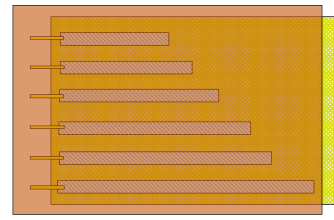

(e) Cross Section
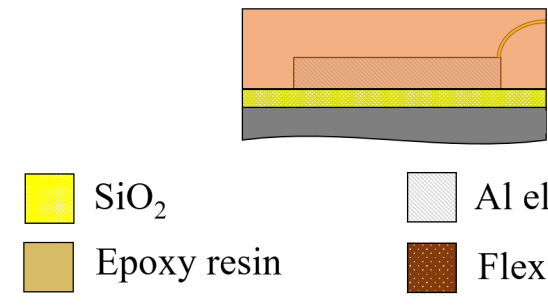

$\mathrm{SiO}_{2}$

Epoxy resin

Au wire bond (b) TS2

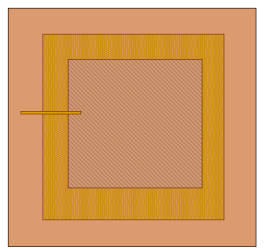

(d) TS4
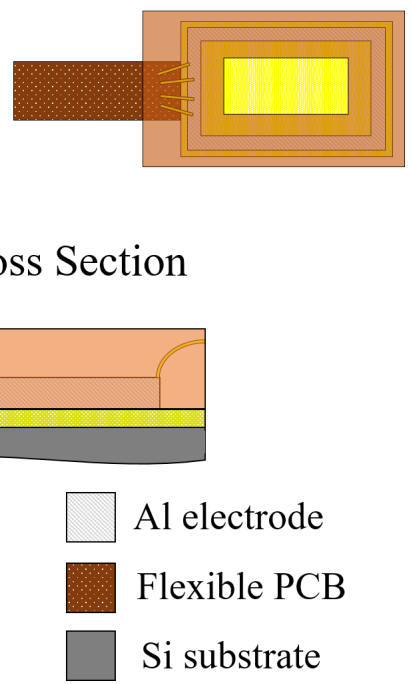

Fig. 1. Top down schematics showing the four test structure designs (a) TS1, (b) TS2, (c) TS3 and (d) TS4. (e) A cross section of the layers in each the test structure designs. Not to scale Not to scale.

This enables the ingress of the liquid environment between the package material/die interface to be monitored, informing both the lifetime of the package and the adhesion of the package material to the die. This measurement is depicted in Fig. 2(c).

4) Test structure four (TS4), as shown in Fig. 1(d), is a $2 \mathrm{~mm}$ by $3 \mathrm{~mm}$ silicon die with a patterned ring of aluminium around the perimeter. This die is then wire bonded to a $1 \mathrm{~mm}$ wide by $20 \mathrm{~mm}$ long flexible printed circuit board (PCB). This set up mimics the final dimensions of an implantable sensor chip, based on the microfabricated sensors developed within the IMPACT project [23]. TS4 enables mock implantation and the electrode can be used to monitor the robustness of the packaging. Fig. 2(d) shows a schematic cross-section of a mock insertion of a TS4 through a biopsy needle.

TS2, 3, and 4 use electrodes to measure leakage current/ion permeation through the material of choice and ingress of liquid solution. This is achieved by applying a potential difference between the electrode under the packaging material and the liquid environment, and monitoring any current. A more detailed explanation and analysis of electrochemical techniques, applied to measure the insulation of barrier materials is reported here [24]. 
(a) TS 1

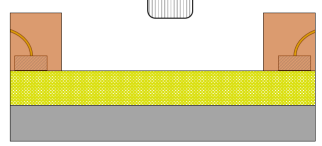

Surface cleanliness of $\mathrm{SiO}_{2}$ after patterning

(ii)

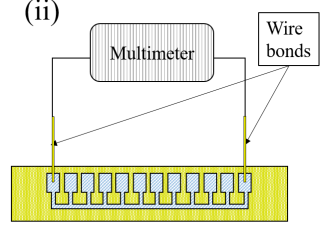

Wire bond continuity (top down view)

(b) TS 2

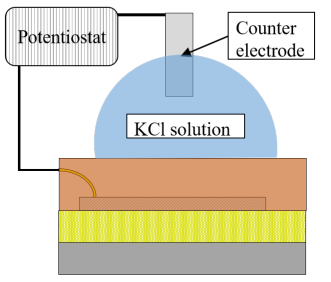

Permeability of packaging material to liquid during packaging process (c) $\mathrm{TS}$

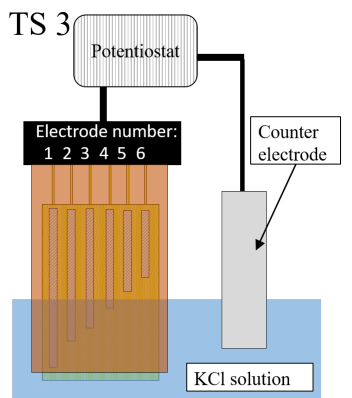

Ingress of liquid at resin/die interface

(d) TS 4

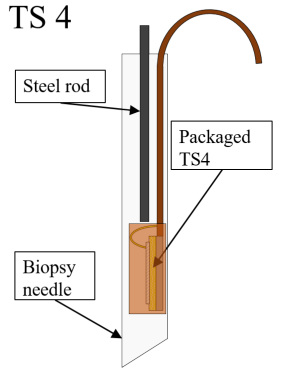

Robustness of package to being implanted through a needle
Fig. 2. Schematic view of each test employed to quantify the packaging material. (a) a (i) cross section through TS1, used monitor surface cleanliness post patterning using a reflectometer and (ii) a top down view of one side of TS1 monitoring wire bond continuity at each stage in the packaging process. (b) A cross section view of TS2 being used to measure how effectively the resin insulates from liquid. (c) TS3 being dipped into solution and used to measure liquid ingress at the resin/die interface through six electrodes with staggered distances and (d) a cross section view of TS4 being inserted through a biopsy needle to assess if the packaging is physically robust. Not to scale.

\section{EXPERIMENTAL SETUP}

\section{A. Fabrication Process}

It is essential that the processes used to fabricate the test structures replicate those used in typical sensor technology [3], [23], [25]. Therefore, for this work, a $500 \mathrm{~nm}$ thick insulating layer of $\mathrm{SiO}_{2}$ is deposited using Plasma Enhanced Chemical Vapour Deposition (PECVD) on a silicon wafer, followed by $1 \mu \mathrm{m}$ of sputtered aluminium. The aluminium is then patterned to form one of the test structures shown in Fig. 1. A schematic cross section of this architecture is shown in Fig. 1(e). As all the text structures used in this work comprise the same layer structure, and hence fabrication process, only one cross section is presented.

\section{B. Test Structure Packaging}

Test structures TS1-TS3 were glued into standard ceramic chip packages, while TS4 was glued to the end of a flexible PCB using Loctite 4014 medical cyanoacrylate adhesive. Wire bonds were made using a HB16 manual Gold Wire Bonder (TPT). The UV sensitive epoxy resin was manually dispensed over the die to fill the cavity of the ceramic package and encapsulate the bond wires. TS1, 3 and 4 were then exposed to UV light through a photomask which shielded areas of the epoxy using a Karl Suss MA/BA 8 mask aligner. As the epoxy resin is a negative type material, the areas shielded from the UV light remained uncured and could easily be removed. These became windows in the resin, exposing the die underneath. For TS1 and TS4, an area in the centre was masked from the UV light and for TS3, the area chosen was a large rectangle which overlapped the die but not enough to expose the metal electrodes. After exposure, the uncured epoxy was removed by first rinsing in acetone, then isopropanol, and finally deionised water before drying with $\mathrm{N}_{2}$.

\section{Measurement Setup}

Electrochemical measurements were carried out using an Autolab PGSTAT 128N potentiostat (Metrohm). A secondary counter/reference electrode was introduced into the solution, which comprised a shard of similarly oxidised silicon wafer coated in $50 \mathrm{~nm}$ of platinum with an underlying $10 \mathrm{~nm}$ titanium adhesion layer. Optical measurements used a Nanospec 3000 reflectometer (Nanometrics) and resistance measurements used a standard digital multimeter (DMM). Flexible PCBs were manufactured by Merlin Flex. For leakage current measurements, a potential difference was applied between the insulated electrodes and the solution. Any measured current above the noise level would indicate solution had infiltrated the packaging and made contact with the electrode. A maximum value of $\pm 5 \mathrm{~V}$ was chosen for most tests, as this is largest potential difference typically required for CMOS (complementary metal oxide semiconductor) electronics. Typically bodily fluid is saline with a salt concentration of roughly around $150 \mathrm{mM}$ [26]. Despite this, a higher salinity measurement solution of $500 \mathrm{mM} \mathrm{KCl}$ was chosen to ensure that, when the insulation failed, the aluminium electrode would corrode providing visual evidence to corroborate the leakage current measurement. All errors are reported as $\pm 3 \sigma$ for repeated measurements of multiple test samples unless stated otherwise.

\section{Measurement Results}

\section{A. Patterning Capability - Surface Quality}

Packaged TS1 structures were first used to determine if the removal of uncured epoxy had been successful. Residual epoxy would not only obscure the electrode surface and impair electrochemical measurements, but could also pose harmful effects to a patient. Therefore, the surface cleanliness was assessed by using an optical reflectometer to measure the thickness of the $\mathrm{SiO}_{2}$ surface before depositing the epoxy and after removing the uncured epoxy, as shown schematically in Fig. 2(a)(i). Since the reflectometer measurement is dependent on the reflective properties of the $\mathrm{SiO}_{2}$, any residual epoxy left on the surface would alter its reflective properties and yield distorted values of $\mathrm{SiO}_{2}$ thickness. A value of $473 \pm 5 \mathrm{~nm}$ was measured before dispensing the resin, followed by a value of $476 \pm 7 \mathrm{~nm}$ after removing the uncured resin. Measurements were made across three chips, each measured in five locations. The data suggests there has been no significant change to 
the $\mathrm{SiO}_{2}$ surface cleanliness, hence that the uncured epoxy has been successfully removed. The slight changes observed before and after can be explained by variability in the locations the measurements were made, and the standard deviation is within the range expected for multiple measurements of blank $\mathrm{SiO}_{2}$ across multiple dies. If epoxy residue had been present then the value measured would have been significantly different to the baseline measurement, potentially even being unable to record a value if a large amount of contamination was on the surface.

\section{B. Patterning Capability - Electrical Continuity}

The connectivity of the electrical connections and wire bonds was determined using TS1, both before dispensing the resin, and after removing the uncured material. Resistance measurements were made on each of the four sides of the TS1 chip and the electrical path measured is shown schematically in Fig. 2(a)(ii). After packaging, all test structures were additionally subjected to 20 minutes of ultrasonic (US) agitation in deionised water to confirm that the cured resin could protect the wire bonds from mechanical damage. The measured resistance before packaging was $11.6 \pm 1.3 \Omega$, and $11.7 \pm 1.8$ $\Omega$ after resin patterning. After applying ultrasonic agitation, the resistance was measured as $11.6 \pm 1.8 \Omega$. These results indicate that the dispensing, curing, and clearing processes have not damaged the wire bonds. The bonds also survived the 20-minute ultrasonic agitation test, which demonstrates the mechanical robustness of the encapsulation material.

\section{Packaging Material Permeability}

Leakage current measurements were performed using TS2 according to the schematic in Fig. 2(b). UV cured glue was manually dispensed to create walls, confining the $\mathrm{KCl}$ solution on top of the resin. A range of voltages were applied between the metal areas on the test structures and the platinum counter electrode immersed in the solution. Any pinholes or permeability of the resin would be indicated by an increase in current. Measurements were performed in a Faraday cage and potentials of $-5,-3,-1.5,0,+1.5,+3$, and $+5 \mathrm{~V}$ were sequentially applied for for 5 minutes each. The current at each potential was measured with an interval time of 0.5 seconds, giving 600 data points per step. No filter was applied to the data. Fig. 3(a) presents the average current measured for each applied potential from three test structures. It is encouraging to see that the currents are on the order of $10 \mathrm{pA}$, on the levels of electrical noise (typically on the order of $10-50$ $\mathrm{pA})$. Although a small positive offset is observable, it unlikely due to leakage through the layer as it is very small (around $10 \mathrm{pA}$ ) and, more crucially, does not change with applied potential. It may originate from the potentiostat circuitry in this particular configuration. As the recorded currents do not change with applied potential, this lends confidence that an open circuit is being measured and hence the conclusion the recorded current stems from background electrical noise rather than ions reaching the metal electrode through pinholes or permeability of the resin. This measurement was repeated after 10 days of immersion in $\mathrm{KCl}$ solution in a humidity-controlled
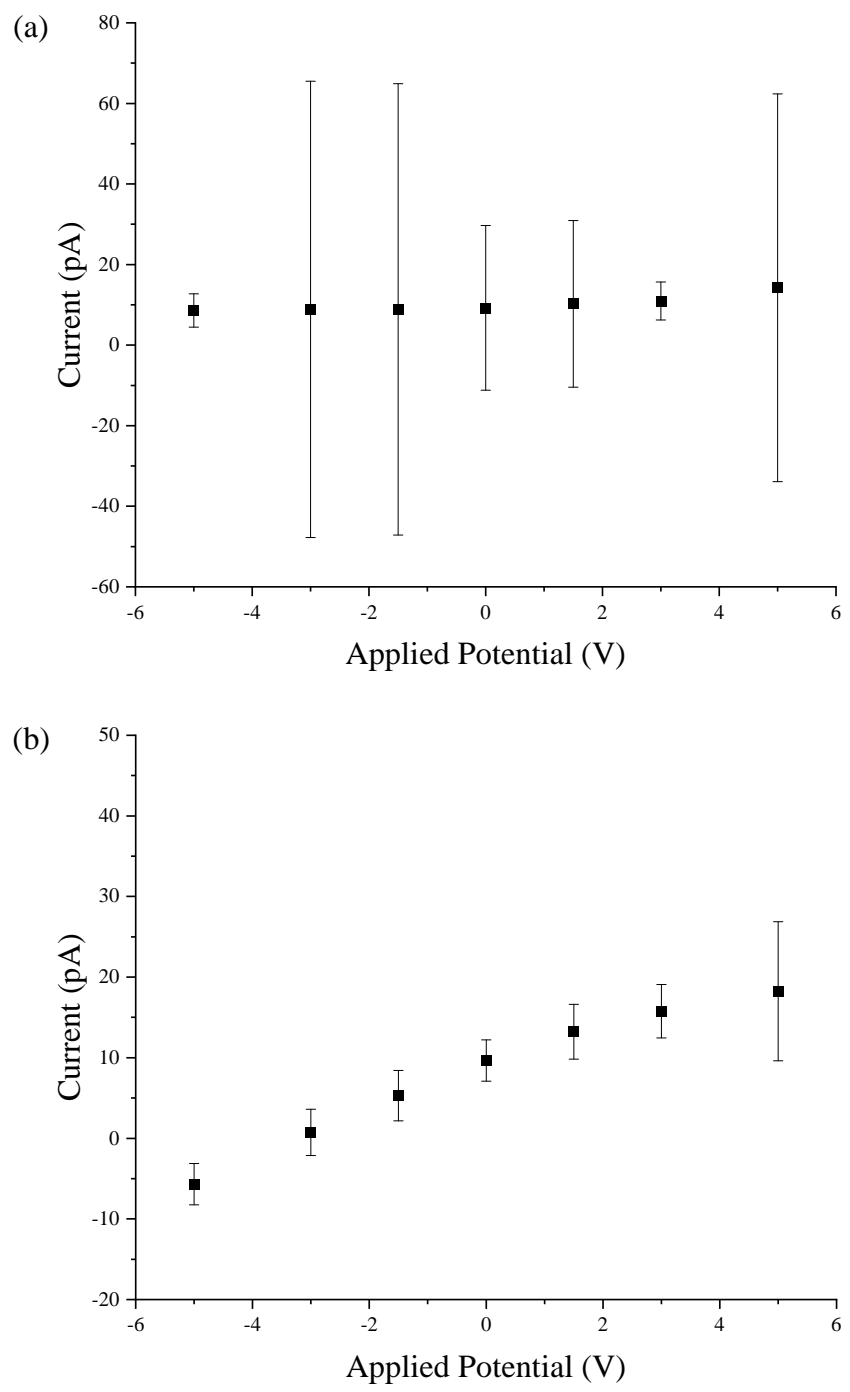

Fig. 3. Plot of average leakage current against applied potential measured on TS2 an (a) three test structures on day one and (b) two test structures on day ten. Error bars for these figures are $\pm 1 \sigma$

chamber. Leakage current from two of the original three test structures still showed currents on the order of picoamperes and their average current is shown in Fig. 3(b), demonstrating the resin is still insulating the die from the solution. However, a trend is present where the average current is changing with applied potential, possibly indicating the beginnings of failure. The third test structure showed increased current and is shown in Fig. 4 where the currents have dramatically increased to the nanoampere scale and also show an obvious linear dependence on potential. This confirms that the test structures can be used to identify instances where the packaging has failed.

\section{Resin Adhesion}

The adhesion of the resin to the die surface was investigated using TS3. The window patterned into the resin overlapped the die, exposing the resin/die interface. Should the liquid environment encroach between the die and resin, it will be measured at the electrodes. The distance between the electrodes and the liquid interface was staggered, so the solution 


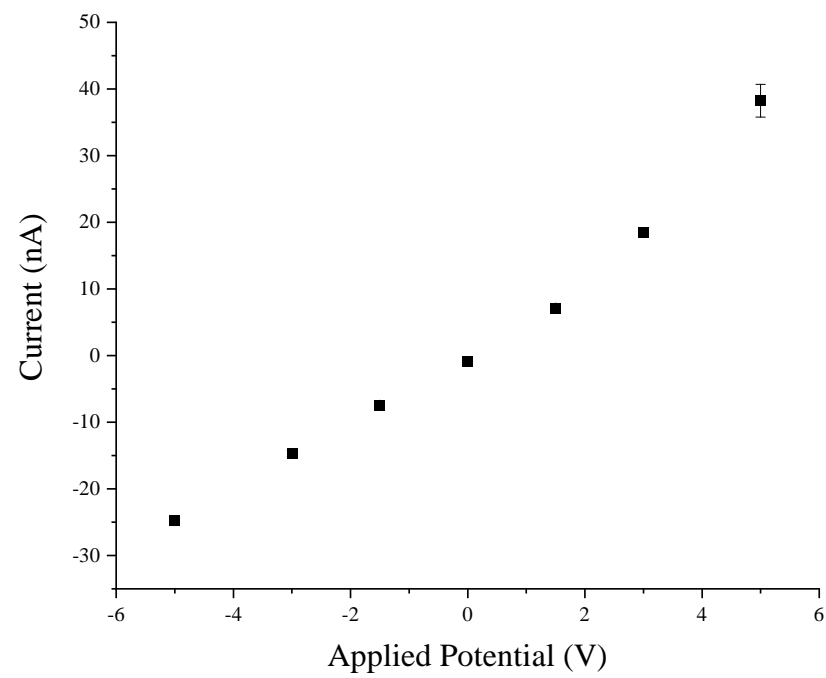

Fig. 4. Plot of average leakage current against applied potential measured on a failed TS2 after 10 days of exposure to $\mathrm{KCl}$ solution. Error bars for these figures are $\pm 1 \sigma$.

would first make contact with the electrode 1 , then electrode 2 , and so on. The time between this occurring at each electrode was divided by the distance between each electrode, giving the rate at which the $\mathrm{KCl}$ progressed between the resin and die surface. Three packages were dipped vertically into $\mathrm{KCl}$ solution until the window patterned in the resin was submerged and the experimental set up is shown schematically in Fig. 2(c). The electrical connections to the electrodes were therefore above the solution, but were still covered with epoxy to prevent any possible shorting. A dc voltage of $+5 \mathrm{~V}$ was applied for ten seconds between the platinum counter electrode and each electrode sequentially over the course of ten days. The experimental set up was too large for the available Faraday cages; hence the background noise was larger than that measured with the TS2 setup. For this reason, the $\mathrm{KCl}$ was deemed to have made contact with the aluminium electrode when the current measured between it and the platinum strip increased to above $10 \mathrm{nA}$. Interestingly, the difference in time between the electrodes making contact with the solution varied greatly, even on the same test structure. Fig. 5 presents a plot from one TS3, where the large increases in current denote the time points at which the $\mathrm{KCl}$ solution reached each electrode. It can be seen that the solution reaches electrode 1 first, followed by electrode 4 , and then electrodes 2 then 3 . The resulting ingress rates ranged from 2 to $48 \mu \mathrm{m} / \mathrm{hour}$, implying that random defects may control the rate of liquid ingress rather than a systematic material fault. It should be noted that only electrodes 1 to 4 were recorded in this measurement due to constraints on the number of channels available on the recording equipment. Fig. 6 presents photographs of a TS3 from (a) before and (b) after 10 days immersion with voltages applied as described above. The aluminium electrodes have clearly been corroded and the there is discolouration, indicating the area where the $\mathrm{KCl}$ solution has ingressed.

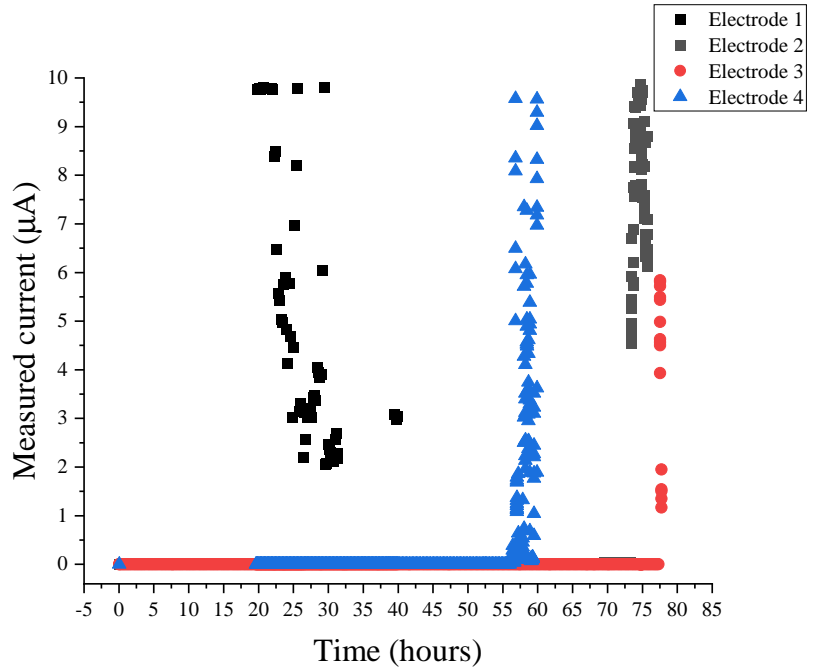

Fig. 5. Plot of current measured over time from a TS3, large increases in current mark the points in time where the $\mathrm{KCl}$ solution made contact with the aluminium electrodes.

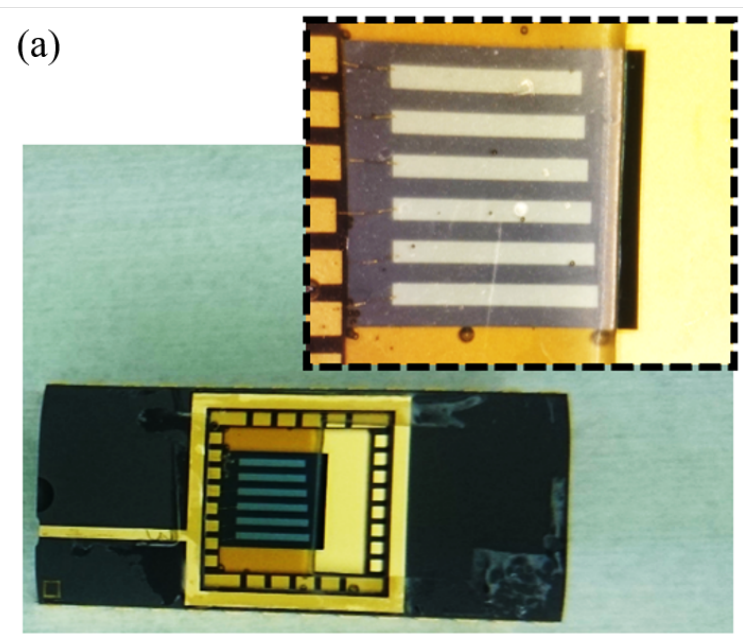

(b)

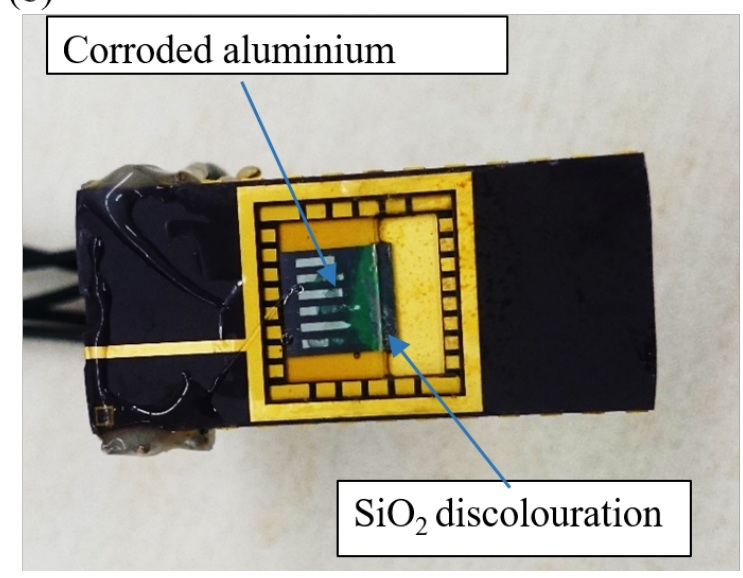

Fig. 6. Photograph of a TS3 (a) after patterning epoxy and (b) after immersion in $\mathrm{KCl}$ at $5 \mathrm{~V}$ for ten days.

\section{E. Implant Capability}

The capability of the IMPACT package design to survive implantation was investigated using TS4. These tests used 
sheep cadavers in order to mimic implantation into the large animal model used for the IMPACT project. The development of the surgical procedure is described elsewhere, but briefly an 8 gauge Jamshidi biopsy needle is advanced to the implant site and the central stylet is removed [27]. The package is fed into the bore of the needle and pushed out into the tissue using a steel rod (obturator), depicted in the schematic in Fig. 2(d). The needle and rod are then withdrawn and the entry point sutured to close the wound and anchor the flexible PCB against the skin. Several insertions were carried out on each test structure. Afterwards optical examination was carried out and very little damage was observed to the package. Fig. 7 shows an instance where damage has occurred at the edge of the package. This is area where the rod pushes the chip into the body and was expected to sustain the worst of any damage. It is gratifying to see the package only sustained superficial damage despite the number insertions performed.

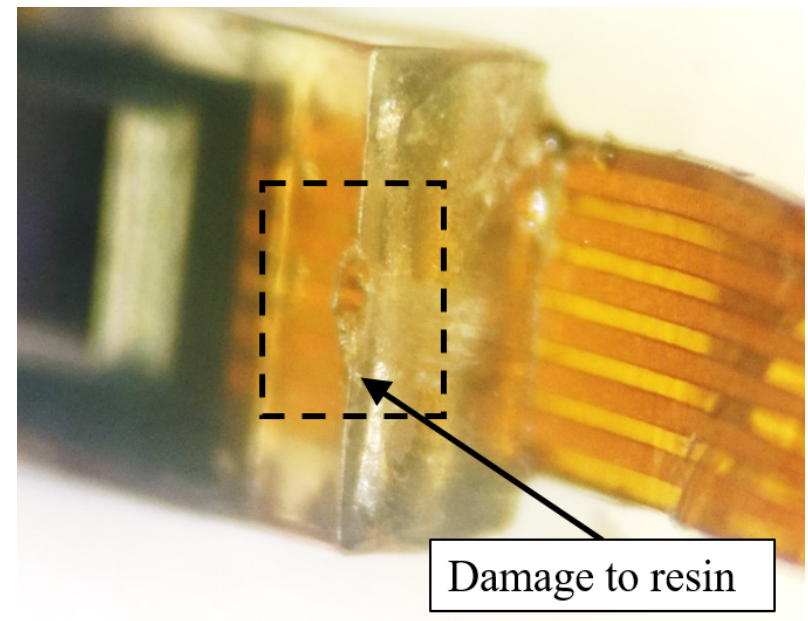

Fig. 7. A photograph of a TS4 after implantation and subsequent removal from a sheep carcass. A small chip can be seen on the top edge and is circled.

\section{Package Optimisation Using Test Structures}

To demonstrate the applicability of these test structures to packaging optimisation, annealing the resin was assessed as a method of improving insulation. Literature around resins and composite materials suggests that thermal annealing could improve the material properties of the resin. This is typically achieved by raising the polymer to near its glass transition temperature, allowing it to rearrange in a more conformal manner and reduce stress [28]. Three TS4s were annealed at $120^{\circ} \mathrm{C}$, slightly above the resin's glass transition temperature of $115^{\circ} \mathrm{C}$ on a closed hotplate for five minutes. They were then immersed in the same $\mathrm{KCl}$ solution and $+5 \mathrm{~V}$ was applied. Fig. 8 presents the current recorded over a period of 80 hours from both annealed and unannealed test structures. It is immediately obvious that the annealed packages tested have a longer lifespan than those that were not. Assuming a leakage current of $1 \mathrm{nA}$ denotes failure, then the annealed packages survived for 45 hours, 45 hours, and 66 hours, while the unannealed lasted for 21 hours and 23 hours. However,
TABLE I

RESISTANCES MEASURED FROM 3 TS1S AFTER EXPOSURE TO DIFFERENT HEAT TREATMENTS.

\begin{tabular}{|c||c|}
\hline Temperature & Resistance $(\Omega)$ \\
\hline Room Temp. & $6.8 \pm 2.3$ \\
$80^{\circ} \mathrm{C}$ & $7.9 \pm 2.8$ \\
$100^{\circ} \mathrm{C}$ & $6.8 \pm 2.2$ \\
$120^{\circ} \mathrm{C}$ & $6.4 \pm 2.5$ \\
$150^{\circ} \mathrm{C}$ & $6.7 \pm 2.7$ \\
\hline
\end{tabular}

as discussed below, although a cutoff such as $1 \mathrm{nA}$ enables quantitative comparison between devices during testing, that threshold may not always be appropriate or realistic. While the variability of responses is high, meaning the anneal step has not improved the variation between different chips, the results suggest that the annealing process could improve the overall performance of the resin. There was also a worry about the stability of the wire bonds as the heat treatment could cause materials in the package to expand, causing damage to the connections. This was hard to assess with TS4, as multiple connections were made to the chips. TS1s therefore underwent a range of heat treatments each for five minutes, before being returned to room temperature, and the wire bond resistances measured as in section IV-B. The results are shown in table I and demonstrate the stability of the wire bonds at all temperatures tested.

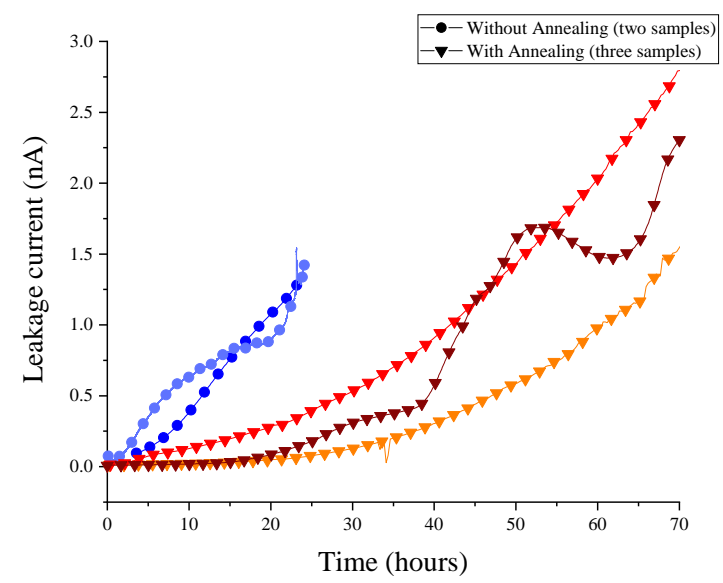

Fig. 8. Plot of leakage current against time of five TS4s held at a potential of $5 \mathrm{~V}$, immersed in $\mathrm{KCl}$ solution. Three devices were annealed (triangles) and two were not (circles).

\section{DISCUSSION}

This work has described a set of test structures aimed at systematically evaluating and optimising packaging for an implantable sensor. The optical measurements of TS1 indicate that the open areas patterned into the resin were free of significant residue, while the results from the electrical measurements demonstrated that the resin is physically robust enough to withstand ultrasonic agitation. This also means harsher cleaning methods could be used if required. Resistance measurements (Tab. I) also showed the electrical connections 
were robust to temperature changes up to $150^{\circ} \mathrm{C}$, enabling the implementation of the anneal step.

The leakage currents measured on TS2 suggest that the resin insulates effectively in $\mathrm{KCl}$. These measurements could be performed using a number of more application-specific aqueous solutions or even at elevated temperatures. Other electrochemical measurement techniques could be employed, such as monitoring open circuit potential. However, applying a significant potential is a more destructive method of assessment, and so it would be expected to accelerate the appearance of any failure [24], [29]. In addition, the use of linearly applying several potentials and measuring the corresponding currents, as opposed to just applying one, may yield more information about the system. For example, in the case of Fig. 3, measuring only one potential before and after the 10 day test would yield a current on the order of picoamperes, which typically marks the package as still insulating. However, when a range of potentials is applied, a change in behaviour can be observed, with a dependence on applied potential emerging in Fig. 3(b) after immersion in $\mathrm{KCl}$ for 10 days, suggesting a change in the insulation properties of the material has occurred.

TS3 enabled a rate of ingress to be determined, although this was highly variable with values ranging between 2 and $48 \mu \mathrm{m} /$ hour. This presented a faster rate of failure than TS2, where only one test structure measured significant current after 10 days, compared with all three TS3s which began measuring current within 24 hours. Ultimately this suggests that liquid ingress between the package and the die surface determines the lifetime of the insulation. The high level of variability points to randomly occurring defects or contaminants affecting the adhesion of the resin to the surface of the die. Adhesion between materials has often been identified as the main cause of failure in packaged sensors and methods such as atomic layer deposition and complex multilayer packaging solutions are often used as a response to this [30], [31]. The test structures presented here and in the literature are a potential strategy for systematically comparing and evaluating these efforts.

TS4 underwent tests to assess the suitability of the packaging for implantation. In line with the results found with TS1, the resin showed good mechanical strength and durability, suffering only superficial damage. Leakage current tests were unfortunately not carried out on TS4 after implantation owing to the build-up of a biological contaminants around the perimeter of the window onto the die surface. This was not easy to clean after drying and could interfere with the lifetime test, as TS3 had identified liquid ingress at this interface as the mechanism of failure.

TS4 was also used to determine the impact of an anneal step during the packaging process. The data suggest that this step successfully increased the lifetime of the insulation. Variability in this response makes determining an average lifetime inappropriate. Another factor in this is the choice of current value at which the device is deemed to have failed. Multiple studies in literature use $1 \mathrm{nA}$ as the cutoff for failed insulation, however this depends on the end application of the sensor. In the case of miniaturised electrochemical sensors, the current response is frequently already on the nanoampere scale, so a lower cut-off would need to be chosen. It is worth noting that lifetime generally decreases with increasing applied potential in these instances and so a sensor operating at a lower voltage will usually demonstrate a higher lifetime [32].

After utilising the test structures depicted here, an optimised packaging system for an implantable sensor would have to be tested in an environment more akin to that of a human body. This would require elevated temperature $\left(37^{\circ} \mathrm{C}\right)$ and leakage tests in media equivalent to that of human bodily fluid (for example, tissue culture medium). Further increasing the temperature and applied potential would accelerate the testing process, but can run the risk of becoming less relevant to the final application.

\section{CONCLUSIONS}

Implantable sensor packaging materials are typically required to insulate effectively in liquid environments, be physically robust enough for implantation, and capable of being patterned. This paper has presented four test structures capable of characterising packaging materials for this application. These test structures were then employed in characterising a biocompatible, UV curable resin.

- TS1 enabled both the successful monitoring of the gold wire bond stability through the packaging process and confirmation that the uncured resin had been removed from the desired areas during patterning;

- TS2 enabled quantification of the resin's permeability to a liquid environment;

- TS3 enabled assessment of the adhesion of the resin to the die surface via monitoring the rate of ingress of the liquid environment between the die and resin and;

- TS4 enabled demonstration the package is robust enough to be implanted.

The combination of these tests result in a comprehensive understanding of the strengths and weaknesses of the selected biocompatible resin. These test structures can then be further used to systematically optimise key parameters of packaging processes and materials. This is not only important for arriving at a successful packaging solution, but when developing medical devices having a systematic trail of development is helpful when engaging with medical regulators. Further work is required to be able to batch test many test structures at once, and for longer durations, in order to assess insulation layer lifetimes. This would enable large scale, statistically significant comparisons of numerous materials. Accelerated testing with elevated temperatures and more aggressive chemical environments may also be desirable to demonstrate long term stability of devices intended for chronic implantation. In addition, studies using sensors packaged in this manner are currently underway [23].

\section{REFERENCES}

[1] M. Gray, J. Meehan, C. Ward, S. P. Langdon, I. H. Kunkler A. Murray, and D. Argyle, "Implantable biosensors and their contribution to the future of precision medicine," The Veterinary Journal, vol. 239, pp. 21 - 29, 2018. [Online]. Available: http: //www.sciencedirect.com/science/article/pii/S1090023318304180 
[2] J. Andreu-Perez, D. R. Leff, H. M. D. Ip, and G. Yang, "From wearable sensors to smart implants--toward pervasive and personalized healthcare," IEEE Transactions on Biomedical Engineering, vol. 62, no. 12 , pp. 2750-2762, 2015.

[3] J. R. K. Marland, E. O. Blair, B. W. Flynn, E. González-Fernández, L. Huang, I. H. Kunkler, S. Smith, M. Staderini, A. Tsiamis, C. Ward, and A. F. Murray, Implantable Microsystems for Personalised Anticancer Therapy. Springer, Cham, 2018, book section 11, pp. pp 259-286.

[4] D. R. Grimes and M. Partridge, "A mechanistic investigation of the oxygen fixation hypothesis and oxygen enhancement ratio," Biomedical physics \& engineering express, vol. 1, no. 4, pp. 045209 045209 , 2015. [Online]. Available: https://pubmed.ncbi.nlm.nih.gov/ 26925254https://www.ncbi.nlm.nih.gov/pmc/articles/PMC4765087/

[5] M. Hoeckel and P. Vaupel, "Tumor hypoxia: Definitions and current clinical, biologic, and molecular aspects," JNCI: Journal of the National Cancer Institute, vol. 93, no. 4, pp. 266-276, 2001. [Online]. Available: https://doi.org/10.1093/jnci/93.4.266

[6] M. W. Dewhirst, Y. Cao, and B. Moeller, "Cycling hypoxia and free radicals regulate angiogenesis and radiotherapy response," Nature Reviews Cancer, vol. 8, no. 6, pp. 425-437, 2008. [Online]. Available: https://doi.org/10.1038/nrc2397

[7] M. Madou, Fundamentals of Microfabrication: The Science of Miniaturization. CRC Press, 2002.

[8] R. Tummala, Fundamentals of Microsystems Packaging. McGraw-Hill Education, 2001.

[9] R. L. Smith and S. D. Collins, "Micromachined packaging for chemical microsensors," IEEE Transactions on Electron Devices, vol. 35, no. 6, pp. 787-792, 1988

[10] Y. Qin, M. M. R. Howlader, M. J. Deen, Y. M. Haddara, and P. R. Selvaganapathy, "Polymer integration for packaging of implantable sensors," Sensors and Actuators B: Chemical, vol. 202, pp. 758 - 778, 2014. [Online]. Available: http://www.sciencedirect.com/science/article/ pii/S0925400514005929

[11] C. Cotofana, A. Bossche, P. Kaldenberg, and J. Mollinger, "Low-cost plastic sensor packaging using the open-window package concept," Sensors and Actuators A: Physical, vol. 67, no. 1, pp. 185 - 190, 1998. [Online]. Available: http://www.sciencedirect.com/science/article/ pii/S0924424797017676

[12] M. A. Miller, P. Neuzil, S. R. Dukkipati, and V. Y. Reddy, "Leadless cardiac pacemakers," Journal of the American College of Cardiology, vol. 66, no. 10, pp. 1179-1189, 2015. [Online]. Available: http://www.onlinejacc.org/content/66/10/1179

[13] R. A. M. Receveur, F. W. Lindemans, and N. F. de Rooij, "Microsystem technologies for implantable applications," Journal of Micromechanics and Microengineering, vol. 17, no. 5, pp. R50-R80, 2007. [Online]. Available: https://doi.org/10.1088\%2F0960-1317\%2F17\%2F5\%2Fr02

[14] J. C. Lötters, W. Olthuis, P. H. Veltink, and P. Bergveld, "The mechanical properties of the rubber elastic polymer polydimethylsiloxane for sensor applications," Journal of Micromechanics and Microengineering, vol. 7, no. 3, pp. 145-147, 1997. [Online]. Available: https: //doi.org/10.1088\%2F0960-1317\%2F7\%2F3\%2F017

[15] J. Munoz, A. Bratov, R. Mas, N. Abramova, C. Dominguez, and J. Bartoli, "Packaging of isfets at the wafer level by photopatternable encapsulant resins," in Proceedings of the International Solid-State Sensors and Actuators Conference - TRANSDUCERS '95, vol. 1, 1995, Conference Proceedings, pp. 248-251.

[16] T. Datta-Chaudhuri, P. Abshire, and E. Smela, "Packaging commercial cmos chips for lab on a chip integration," Lab Chip, vol. 14, pp. 17531766, 2014. [Online]. Available: http://dx.doi.org/10.1039/C4LC00135D

[17] G. Jiang and D. D. Zhou, Technology Advances and Challenges in Hermetic Packaging for Implantable Medical Devices. New York, NY: Springer New York, 2010, pp. 27-61. [Online]. Available: https://doi.org/10.1007/978-0-387-98120-8_2

[18] K. Najafi, "Packaging of implantable microsystems," in SENSORS, 2007 IEEE, 2007, Conference Proceedings, pp. 58-63.

[19] A. J. Walton, "Microelectronic test structures," in Semicon '97, 1997, Conference Proceedings.

[20] N. Abramova and A. Bratov, "Photocurable polymers for ion selective field effect transistors. 20 years of applications," Sensors (Basel), vol. vol 9, no. 9, pp. 7097-7110, 2009.

[21] E. O. Blair, A. Buchoux, A. Tsiamis, C. Dunare, J. R. K. Marland, J. G. Terry, S. Smith, and A. J. Walton, "Test structures for the characterisation of sensor packaging technology," in 2017 International Conference of Microelectronic Test Structures (ICMTS), 2017, Conference Proceedings, pp. 1-6.

[22] M. E. Gray, J. Meehan, E. O. Blair, C. Ward, S. P. Langdon, L. R. Morrison, J. R. K. Marland, A. Tsiamis, I. H. Kunkler,
A. Murray, and D. Argyle, "Biocompatibility of common implantable sensor materials in a tumor xenograft model," Journal of Biomedical Materials Research Part B: Applied Biomaterials, vol. 107, no. 5, pp. 1620-1633, 2019. [Online]. Available: https://onlinelibrary.wiley.com/ doi/abs/10.1002/jbm.b.34254

[23] M. E. Gray, J. R. K. Marland, C. Dunare, E. O. Blair, J. Meehan, A. Tsiamis, I. H. Kunkler, A. F. Murray, D. Argyle, A. Dyson, M. Singer, and M. A. Potter, "In vivo validation of a miniaturized electrochemical oxygen sensor for measuring intestinal oxygen tension," American Journal of Physiology-Gastrointestinal and Liver Physiology, vol. 317, no. 2, pp. G242-G252, 2019. [Online]. Available: https://doi.org/10.1152/ajpgi.00050.2019

[24] G. Bitko, D. J. Monk, T. Maudie, D. Stanerson, J. Wertz, J. Matkin, and S. Petrovic, "Analytical techniques for examining reliability and failure mechanisms of barrier-coated encapsulated silicon pressure sensors exposed to harsh media," in Micromachined Devices and Components II, K. H. Chau and R. M. Roop, Eds., vol. 2882. SPIE, 1996, Conference Proceedings, pp. 248 - 258. [Online]. Available: https://doi.org/10.1117/12.250710

[25] S. Libertino, S. Conoci, A. Scandurra, and C. Spinella, "Biosensor integration on si-based devices: Feasibility studies and examples," Sensors and Actuators B: Chemical, vol. 179, pp. $240-251$, 2013. [Online]. Available: http://www.sciencedirect.com/science/article/ pii/S0925400512010222

[26] E. L. F. P. F. M. S. R. Rossana Berardi, Mariangela Torniai, "Electrolyte disorders in cancer patients: a systematic review," Journal of Cancer Metastasis and Treatment, 2019.

[27] M. E. Gray, P. Sullivan, J. R. K. Marland, S. N. Greenhalgh, J. Meehan, R. Gregson, R. E. Clutton, C. Cousens, D. J. Griffiths, A. Murray, and D. Argyle, "A novel translational ovine pulmonary adenocarcinoma model for human lung cancer," Frontiers in Oncology, vol. 9, no. 534, 2019. [Online]. Available: https: //www.frontiersin.org/article/10.3389/fonc.2019.00534

[28] A. J. de Gee, P. Pallav, A. Werner, and C. L. Davidson, "Annealing as a mechanism of increasing wear resistance of composites," Dental Materials, vol. 6, no. 4, pp. 266-270, 1990. [Online]. Available: http://www.sciencedirect.com/science/article/pii/S0109564105800089

[29] B. S. Skerry and D. A. Eden, "Characterisation of coatings performance using electrochemical noise analysis," Progress in Organic Coatings, vol. 19, no. 4, pp. 379 - 396, 1991. [Online]. Available: http://www.sciencedirect.com/science/article/pii/003306559180019F

[30] R. Caldwell, H. Mandal, R. Sharma, F. Solzbacher, P. Tathireddy, and L. Rieth, "Analysis of al2o3-parylene c bilayer coatings and impact of microelectrode topography on long term stability of implantable neural arrays," Journal of Neural Engineering, vol. 14, no. 4, p. 046011, 2017. [Online]. Available: http://dx.doi.org/10.1088/1741-2552/aa69d3

[31] C. Li, M. Cauwe, Y. Yang, D. Schaubroeck, L. Mader, and M. Op de Beeck, "Ultra-long-term reliable encapsulation using an atomic layer deposited hfo2/al2o3/hfo2 triple-interlayer for biomedical implants," Coatings, vol. 9, no. 9, p. 579, 2019. [Online]. Available: https://www.mdpi.com/2079-6412/9/9/579

[32] X. Chen and D. Young, "Robust implantable blood pressure sensor packaging for long-term laboratory animals monitoring," in 2016 IEEE SENSORS, 2016, Conference Proceedings, pp. 1-3. 